TITLE:

\title{
Mechanism and control of periodic surface nanostructure formation with femtosecond laser pulses
}

$\operatorname{AUTHOR}(S)$ :

Miyazaki, Kenzo; Miyaji, Godai

\section{CITATION:}

Miyazaki, Kenzo ...[et al]. Mechanism and control of periodic surface nanostructure formation with femtosecond laser pulses. Applied Physics A 2013, 114(1): 177-185

\section{ISSUE DATE:}

2013-11-16

URL:

http://hdl.handle.net/2433/196857

\section{RIGHT:}

The final publication is available at Springer via http://dx.doi.org/10.1007/s00339-0138130-4; This is not the published version. Please cite only the published version.; この論文 は出版社版でありません。引用の際には出版社版をご確認ご利用ください。 


\title{
Mechanism and control of periodic surface nanostructure formation with femtosecond laser pulses
}

\author{
Kenzo Miyazaki • Godai Miyaji
}

Received :

Fundamental mechanism of femtosecond (fs) laser-induced periodic surface nanostructure formation has been investigated under the condition using superimposed multiple shots at lower fluence than the single-pulse ablation threshold. With increasing the shot number of low-fluence fs laser pulses, the periodic nanostructure develops through the bonding structure change of target material, the nanoscale ablation with optical near-fields induced around the high curvatures, and the excitation of surface plasmon polaritons (SPPs) to create the nano-periodicity in the surface structure. It is confirmed that non-thermal interaction at the surface plays the crucial role in the nanostructure formation. Based on the mechanism, we have demonstrated that the periodic nanostructure formation process can be controlled to fabricate a homogeneous nanograting on the target surface, using a two-step ablation process in air. The experimental results obtained represent exactly the nature of a single spatial standing SPP wave mode that generates periodically enhanced near-fields for the nanograting formation. The calculated results for a model target reproduce well the nanograting period and explain the characteristic properties observed in the experiment.

K. Miyazaki (corresponding author) • G. Miyaji

Laser Science Research Section, Institute of Advanced Energy, Kyoto University, Gokasho, Uji, Kyoto, 611-0011, Japan

e-mail:miyazaki@iae.kyoto-u.ac.jp 


\section{Introduction}

Intense femtosecond (fs) laser pulses have been used as the common tool for precision processing of materials, since the ultrafast energy deposition can minimize undesirable thermal and mechanical effects [1,2]. In the fs-laser ablation experiment, spontaneous self-organization process has often been observed to produce nanoscale periodic structures on the surface of target materials such as dielectrics [3-6], semiconductors [7-9] and metals [10-12]. The observed nanostructure size, typically $1 / 10-1 / 5$ of the laser wavelength $\lambda$, has attracted considerable interest, because the self-organized nanostructure suggests a potential route to circumvent the fundamental limit of spatial resolution due to diffraction in the conventional laser processing.

Formation of similar laser-induced periodic surface structures with a size on the order of $\sim \lambda$ has long been known as a universal phenomenon, so-called ripples. The ripple formation has traditionally been explained by the interference between the incident light and a surface electromagnetic wave $[13,14]$. Recent studies take into account additional processes such as surface plasmons and thermal effects to illustrate the formation of sub-wavelength ripples [15-17]. The ripple formation mechanisms, however, can never explain the origin of fs-laser-induced nanostructures much smaller than $\lambda$.

A number of interaction processes have been proposed so far to explain the mechanism of fs-laser-induced nanostructuring. Those are concerned with self-organization of surface instability $[3,18]$, linear and/or nonlinear refractive index change [5,9], Coulomb explosion [6], second-harmonic generation [7,19], nanoplasma formation [20,21], optical near-fields [22,23] and excitation of surface plasmon polaritons (SPPs) in the surface layer [24,25]. Some of these mechanisms should contribute to the observed periodic nanostructure formation in specific cases. Despite the extensive studies, however, the underlying physics of periodic nanostructure formation is still insufficiently understood, and the experimental demonstration of nanostructuring mechanism and/or its control remain challenging.

In the experimental study of fs-laser-induced nanostructure formation, we have focused our attention on the experimental condition of nanostructuring that uses superimposed multiple shots of fs laser pulses at lower fluence than the single-pulse ablation threshold of the target [22-26]. This condition has extensively been employed to observe the formation of periodic nanostructures for a variety of materials [3-12]. Under the condition, the fs laser pulses are expected to hardly induce strong thermal effects such as thermal melting and 
erosion before the onset of ablation [26-28], and then the ablation trace should be the fingerprint of ultrafast interaction of fs laser pulses with the target surface.

In this paper we present a review of our recent experimental studies on the mechanism of periodic surface nanostructure formation under the condition of interest. The experimental results have shown that the periodically enhanced near-fields of SPPs are predominantly responsible for the formation of periodic surface nanostructures [24,25]. Based on the mechanism, we demonstrate that the excitation of SPPs can be controlled to fabricate a homogeneous nanograting on solid surface in air $[29,30]$.

Here the periodic nanostructure is referred to as the ablated surface structures with the period size less than $\sim \lambda / 2$, whereas the larger structure may be identified as ripples. In the next section, we describe the experimental procedure. Section 3 summarizes the fundamental processes for the formation of periodic surface nanostructures, based on the results obtained in our experiments. Section 4 presents the experimental study on the role of multiple low-fluence fs laser pulses in nanostructuring, and in Sec. 5 we describe the experiment to control the nanostructure formation process and fabricate a homogeneous nanograting on the target surface.

\section{Experimental}

In the experiments we used a Ti:sapphire fs-laser system that produced linearly-polarized $800 \mathrm{~nm}, 100$-fs pulses at a repetition rate of $10 \mathrm{~Hz}$. The output had a well-defined lowest-order Gaussian spatial distribution of intensity, and its pulse-to-pulse fluctuation was minimized for the study of nanostructuring. The peak fluence $F$ on the target surface was precisely controlled with a pair of wave plate and polarizer. Throughout the experiments, $F$ for nanostructuring was less than the single-pulse ablation threshold $F_{1}$ of each target material. The laser polarization was controlled with half- and quarter-wave plates. As well as $F$, one of the most important parameters for nanostructuring was the superimposed shot number $N$ of fs laser pulses on the target surface. The ambient material for the target was also changed when it appeared to have an effect on nanostructuring.

The ablated surface was observed with a scanning electron microscope (SEM) and a scanning probe microscope (SPM). With the two-dimensional Fourier transform we analyzed the SEM or SPM images to see the distribution of period in the ablated surface structure. 


\section{Fundamental processes}

Under the condition of interest, we observed morphological change of target surface as a function of $N$ or $F$ to see the development of nanostructure formation [22-29]. The results obtained for different materials have shown that the periodic surface nanostructure formation on the flat surface develops through the processes initiating the nanoscale ablation and growing the nano-periodicity in the ablation trace.

Figure 1 shows a typical example of the SEM images representing the evolution of diamond-like carbon (DLC) surface structure with increasing $N$ at a low fluence [24], where the schematic under the image provides a sketch of the interaction process at each stage [31]:

(a) Bonding structure change: A single fs-laser pulse at $F<F_{1}$ never ablates the target surface under the condition of interest. However, the low-fluence fs laser pulses are intense enough to produce free electrons through ionization of the target material and induce bonding structure change $[27,32]$ in the surface layer. The bonding structure change has been found to play important roles in the initial stage of nanostructure formation [24-27], which should be a non-thermal process, such as electronic melting [33,34], to modify the initial lattice structure. On the DLC surface, the bonding structure change brings about nanoscale swelling of the flat surface and leads to the random nanoablation with optical near-fields enhanced around the high curvatures [22,27,32]. As seen on the SEM image in Fig. 1(a), the initial ablation represents no polarization-dependent periodicity in the ablation trace.

Accumulation of the bonding structure change with increasing $N$ leads to a decrease in the ablation threshold $F_{\text {th }}$, through the formation of a thin layer with a modified optical property, which might be identified as the incubation effect induced at $F<F_{1}$ [27,35]. In fact we have observed such a decrease in $F_{\text {th }}$ for different target materials.

(b) Near-field ablation: As seen in Fig. 1(b), the successive near-filed ablation with increasing $N$ starts to create a nanoscale periodicity in the ablation trace, since the optical near-fields are directed along the laser polarization. The important role of near-fields in nanostructuring has been demonstrated in the experiment using the patterned DLC target [23], where the periodic nanoablation was preferentially initiated at high curvature on the crest of stripes in the direction perpendicular to the laser polarization.

(c) Excitation of SPPS: As the target surface with nanoscale periodicities is ionized with fs laser pulses, the incient $E$-field tends to coherently couple with the collective oscillation of free electrons to excite SPPs [36]. The periodically enhanced near-fields of SPPs should form the periodic nanostructure through the ablation of surface layer, as shown in Fig. 1(c). 
When the excited area is limited on the target surface, one may expect the development of spatial standing SPP wave modes along the laser polarization [36,37]. To simulate the nanostructuring process, we note the possible excitation of SPPs at the interfaces between the thin metalized layer and the dielectric ambient material or the substrate. The calculation has reproduced well the nanoscale periodicity observed for different materials $[24,25,29]$.

The experimental results obtained have also shown that the interaction processes for nanostructuring are very sensitive to the target material, its surface condition, and the ambient material, as well as the laser parameters, whereas those are closely related with each other and often competing with thermal or dynamic processes to disturb the nanostructure formation.

\section{Role of multiple shots at low fluence}

In the nanostructure formation, the incident laser pulse energy localizes in the target material to induce direct solid-vapor or -plasma transition followed by the nanoablation [38]. Since the ablation is delayed by several ps $-100 \mathrm{ps}$ from the fs pump interaction [1,2], non-thermal interaction is crucial to maintain the nanoscale energy distribution in the surface layer until the onset of ablation. To confirm this we measured time-dependent change in the reflectivity $R$ of polished crystalline $\mathrm{Si}$ (c-Si) immersed in water, using a pump and probe technique [28]. The water environment was necessary, because the fine nanostructure with $d \sim 150 \mathrm{~nm}$ observed on $\mathrm{Si}$ in water was never formed in air [25], due most likely to the thermal effect.

The optical configuration for the pump-probe experiment is shown in Fig. 2 (a), where the $p$-polarized pump beam is obliquely incident, while the orthogonally polarized probe pulse with the time delay $\Delta t$ is incident at normal to cover the whole pumped area. Every shot of the reflected probe pulse was recorded with a CCD camera, and the reflectivity $R(\Delta t)$ was measured for the central pump beam area of $40 \mu \mathrm{m}$ in diameter.

Figure 2(b) shows the temporal change in $\eta=R / R_{0}$ for a single pump pulse at $F=120$ $\mathrm{mJ} / \mathrm{cm}^{2}$, where $R_{0}=22 \%$ is the original reflectivity of c-Si surface in water. The pump pulse is absorbed by bound electrons in the surface layer, followed by the ionization to rapidly increase the free-electron density $N_{\mathrm{e}}$ in the surface layer, which induces a rapid decrease of $\eta$ to $\sim 0.8$ at $\Delta t \sim 0.5$ ps. After the end of interaction, $\eta$ slowly decays due primarily to the cooling down of free electrons through the energy transfer to the lattice [39]. The time scale of this electron relaxation is known to be of the order of ps. Using the Drude expression, the electron density $N_{\mathrm{e}}$ is estimated as $N_{\mathrm{e}} \sim 1.0 \times 10^{21} \mathrm{~cm}^{-3}$ from the minimum 
value of $\eta$ at $\Delta t \sim 0.5 \mathrm{ps}$.

Keeping $\Delta t=0.5 \mathrm{ps}$, we measured $\eta$ as a function of $N$ at the same fluence, and the results are shown in Fig. 2(c). With increasing $N, \eta \sim 0.8$ is almost constant up to $N \sim 600$, while no morphological change was observed on the surface. However, $\eta$ rapidly grows up to $\eta_{\max } \sim 1.2$ at $N \sim 700$, indicating the onset of ablation. With a further increase in $N, \eta$ monotonously decreases most likely due to the increase in absorption and scattering on the surface. We observed the formation of a fine periodic nanostructure with $d \sim 150$ at $N \sim$ 1000 , which was preceded by the formation of a course structure with $d \sim 400 \mathrm{~nm}$ [25].

It is clearly shown in Fig. 2(c) that $F_{\text {th }}$ decreases with an increase in $N$. This takes place due to the bonding structure change from c-Si to amorphous $\mathrm{Si}(\mathrm{a}-\mathrm{Si})$ in the region of $N<\sim$ 700. We confirmed this modification to a-Si with the Raman spectra. With an increase in the density of a-Si, $N_{\mathrm{e}}$ in the surface layer increases to bring about the onset of ablation or the abrupt enhancement of $\eta$ to $\eta_{\max } \sim 1.2$ at $N \sim 700$. The electron density estimated from $\eta_{\max }$ is $N_{\mathrm{e}}=(0.6-1.0) \times 10^{22} \mathrm{~cm}^{-3}$. This value is consistent with $N_{\mathrm{e}}$ for the possible excitation of SPPs in our previous study for $\mathrm{Si}[25]$.

To see the thermal effect on the fine nanostructure formation, we measured $\eta_{\max }$ at the different time delay $\Delta t$ at $F_{\text {low }}=120 \mathrm{~mJ} / \mathrm{cm}^{2}$ and $F_{\text {high }}=160 \mathrm{~mJ} / \mathrm{cm}^{2}$, where $F_{\text {high }}$ could never produce the fine nanostrutrure. The peak values $\eta_{\max }$ observed are plotted as a function of $\Delta t$ in Fig. 3. At $F_{\text {low }}, \eta_{\max } \sim 1.2$ is almost constant at $\Delta t=0.5-50$ ps, while $\eta_{\max }$ at $F_{\text {high }}$ increases up to $1.3-1.4$ at $\Delta t=2-50 \mathrm{ps}$. In the region of $\Delta t>\sim 20 \mathrm{ps,} \eta_{\max }$ would be dominated with the surface temperature $T_{\mathrm{s}}$ [40]. We estimated $T_{\mathrm{s}}$ from $\eta_{\max }$ at $\Delta t=50 \mathrm{ps}$. The result have shown that $\eta_{\max }$ at $F_{\text {low }}$ provides $T_{\mathrm{s}}=1200-1300 \mathrm{~K}$, while $\eta_{\max } \sim 1.38$ at $F_{\text {high }}$ exceeds $\eta \sim 1.35$ at the melting point $T_{\text {melt }}=1420 \mathrm{~K}$ of a-Si. This explains the reason why $F_{\text {high }}$ never produces the fine periodic nanostructure. The thermal melting of a-Si layer should destroy the fine energy distribution created at the surface. Thus the multiple shots of low fluence are crucial to maintain $T_{\mathrm{s}}<T_{\text {melt }}$ in the periodic fine nanostructure formation on the target.

\section{Control of nanostructuring}

The self-organized periodic nanostructures are usually inhomogeneous, as seen in Fig. 1. We have shown that, based on the mechanism discussed above, the interaction process for nanostructuring can be controlled to regulate the periodicity in the surface structures $[29,30]$. 
The experiment was made for polished crystalline gallium nitride $(\mathrm{GaN})$, which is transparent at $\lambda \sim 800 \mathrm{~nm}$ but can ionize through three-photon absorption.

In the preliminary experiment, we measured the single-pulse ablation threshold $F_{1}=800$ $( \pm 10) \mathrm{mJ} / \mathrm{cm}^{2}$ of the $\mathrm{GaN}$ to compare $F_{\text {th }}$ for self-organized nanostructure formation with multiple pulses. Figure 4 shows SEM images of the periodic nanostructure formed with $N=$ 100 at (a) $F=400 \mathrm{~mJ} / \mathrm{cm}^{2}$ and (b) $F=480 \mathrm{~mJ} / \mathrm{cm}^{2}$, together with the distributions of the period $d$ in the surface structure. It is clearly seen that the superimposed 100 shots would increase the bonding structure change or defect sites to decrease $F_{\text {th }}$ to $0.5 F_{1}-0.6 F_{1}$, as discussed above.

\subsection{Two-step ablation process}

The self-organized periodic nanostructures shown in Fig. 4 include the non-uniform periods in a range of $d=150-280 \mathrm{~nm}$. We designed a two-step ablation process to control the nanostructure formation process and form a homogeneous nanograting on the GaN surface. In the experiment, the fs laser output was split into two beams (Beam 1 and Beam 2). Both beams with horizontal polarization were focused on the target surface, where Beam 1 was incident at normal while Beam 2 was obliquely incident on the target at the relative angle $\theta$, as shown in Fig. 5(a). The pulse energy or $F$ of each beam was independently controlled with a pair of half-wave plate and polarizer.

In the first step, Beam 1 and 2 were overlapped in space and time to form an interference pattern with the fringe period $\Lambda=\lambda / \sin \theta$ on the target. The target surface was ablated with a single shot of fs pulse at $F=340-400 \mathrm{~mJ} / \mathrm{cm}^{2}$ to create an interference pattern with fringes in the direction perpendicular to the laser polarization. Figure 5(b) shows the SPM image of the interference pattern with $\Lambda=937 \mathrm{~nm}$ produced at $\theta=59^{\circ}$.

In the second step, subsequent to the first step, the fringe pattern was irradiated with multiple shots of fs laser pulses at $F<F_{1}$ from Beam 1, while Beam 2 was blocked out in the optical arrangement. As discussed in Sec. 3, the multiple shots of low-fluence fs laser pulses are expected to reduce the period $\Lambda$ and form a nanograting through the excitation of SPPs on the patterned surface.

\subsection{Nanograting formation}


The nanograting formation in the second step was successfully achieved with $N=30-$ 40 at $F=320-480 \mathrm{~mJ} / \mathrm{cm}^{2}$ from Beam 1. Figure 6 shows a pair of the SEM image of ablated GaN surface and its Fourier spectrum observed as a function of $N$ at $F=400 \mathrm{~mJ} / \mathrm{cm}^{2}$, where each image was observed through the two-step process on the different surface area of a target. The bright and dark stripes in the SEM image correspond to the ridges and the grooves in the structure, respectively. The initial interference pattern for $N=0$ represents a frequency peak at $1 / \Lambda=1.07 \mu \mathrm{m}^{-1}$ or $\Lambda=937 \mathrm{~nm}$, where the other spectral peaks at the harmonic frequencies are virtual. After $N=10$, the surface image represents parallel dark lines of ablation traces at both edges of the initial groove, while no strong ablation takes place on the ridge. This preferential ablation on the groove is due to the reduced ablation threshold, as the results of defect sites created in the first step and near-fields enhanced at the high-curvature along the groove edge. With increasing $N$ up to 40 , the surface structure is a homogeneous nanograting with a uniform period of $d=187 \mathrm{~nm}$, as also seen with the isolated spectral peak in Fig. 6 (d).

Comparing with $N=100$ for the results shown in Fig. 4, we notice that the enhanced near-fields on the initial fringe pattern play an essential role in decreasing $N$ for the nanograting formation. Furthermore, we note in Fig. 6 that the nanograting is formed at $d \sim$ $\Lambda / q$ with $q=5$. It is clear that the reduction of $\Lambda$ is non-thermally achieved with an increase in $N$, while the interference pattern is never destroyed. These results strongly suggest that the ablation to reduce $\Lambda$ is induced by the spatial standing SPP waves excited in the period $\Lambda$, and a single standing wave mode survives with increasing $N$ to form the homogeneous nanograting with $q=5$. The origin of integer $q$ will be identified by a model calculation.

We observed the cross-sectional shape of nanogratings with a scanning transmission electron microscope (STEM). Figures 7 shows the STEM image (lower) of the nanograting (upper SPM image), which is the same as in Fig. 6(d). The height of protrusions in the periodic structure is in a range of $h=530-730 \mathrm{~nm}$. Whereas $h$ is not uniform, it is noted that the bottom of valleys is deeply ablated at a uniform period on a smooth horizontal level. This indicates that intense local fields having the uniform peak intensity are periodically created to ablate the valley bottom.

The electron diffraction patterns observed for the inside of protrusions and the valley bottom represented the same crystalline structure and orientation as those of the non-irradiated GaN substrate. This indicates that the periodic ablation took place at a sharp boundary of the valley bottom, inducing neither appreciable density of defects nor damage in 
the residual substrate. On the other hand, the head of protrusions was capped with a thin amorphous layer of less than $10 \mathrm{~nm}$. The amorphousization would be induced with the defect sites of which density increased with an increase in $N$, while the random ablation of the amorphous layer at the head should create the non-uniform height $h$ seen in Fig. 7(b).

We have found that the nanograting period $d \sim \Lambda / q$ discretely changes with a different integer $q$ when a higher fluence is used to downsize the interference pattern with $\Lambda=937 \mathrm{~nm}$. Figure 8 shows the SEM image of ablated surface and its Fourier spectrum observed with $N=$ 10 and 30 at $F=480 \mathrm{~mJ} / \mathrm{cm}^{2}$. For $N=10$, the narrow line-like ablation traces at $\sim \Lambda / 5$ are predominantly produced on the groove. After $N=30$, a homogeneous nanograting is formed with $d \sim \Lambda / 4=234 \mathrm{~nm}$, while the other peaks are greatly suppressed. The discrete change to $d \sim \Lambda / 4$ from $\Lambda / 5$ observed at $F=400 \mathrm{~mJ} / \mathrm{cm}^{2}$ is also the nature of a spatial standing SPP wave mode formed in the period $\Lambda$.

The downsizing process due to the standing SPP waves was further confirmed for the target with a larger fringe period $\Lambda \sim 1610 \mathrm{~nm}$, which was structured at $\theta=30^{\circ}$ in the first step. The nanograting periods observed in the second step were $d=232 \mathrm{~nm}$ or $268 \mathrm{~nm}$, corresponding to $q=7$ or 6 , respectively. The distribution of $d$ often included sub-peaks for $q=8$, 9, or 10 . These results show that multiple modes of standing SPP waves are simultaneously excited in the larger fringe period.

\subsection{Origin of nanograting periods}

The origin of $d$ or the integer $q$ in the second step was identified with a calculation of the SPP wavelength $\lambda_{\text {spp. }}$ The model surface for calculation is illustrated in the inset of Fig. 9, where we assume that the fs laser pulse (arrow) incident on the target in air (layer $a$ ) forms a thin excited layer (layer $b$ ) on the substrate (layer $c$ ). The interference pattern created in the first step allows the coherent coupling of incident fs laser pulses with SPPs at the interfaces $a / b$ and/or $b / c$, as the layer $b$ is metalized through the ionization.

The method of calculation is the same as those in our previous studies [24,25]. We used the dielectric constants $\varepsilon_{\mathrm{GaN}}=5.3+i 0.0015$ for GaN [41] and $\varepsilon^{*}=\varepsilon_{\mathrm{GaN}}-\left[\omega_{\mathrm{p}}{ }^{2} /\left(\omega^{2}+i \omega / \tau\right)\right]$ for the excited layer $b$, with the laser frequency $\omega$ in vacuum, the Drude damping time of free electrons $\tau\left(=1 \mathrm{fs}\right.$ ) [42], and the plasma frequency $\omega_{\mathrm{p}}$ at $N_{\mathrm{e}}$ and the optical effective mass of carriers $m^{*}=0.2[43]$.

Figure 9 shows the calculated results of $D=\lambda_{\mathrm{spp}} / 2$ as a function of $N_{\mathrm{e}}$ for the interfaces 
$a / b$ and $b / c$, since the ablation traces would predominantly be produced at every half period of a standing SPP wave. The excitation of SPPs is allowed in the region of $\varepsilon^{*}<0$ with $N_{\mathrm{e}}>$ $1.7 \times 10^{21} \mathrm{~cm}^{-3}$, where the calculated results are given by the solid curves. The large period for the interface $a / b$ might correspond to $d$ of sub-wavelength ripples $[15,16]$ that should be formed under different experimental conditions. The small period $D=150-320 \mathrm{~nm}$ for the interface $b / c$ is in good agreement with those of nanogratings observed in the experiment. This result of $D$ indicates that the nanograting period $d=\Lambda / q$ to be created is limited in the range of $150 \mathrm{~nm}<\Lambda / q<320 \mathrm{~nm}$, leading to $q=4$ or 5 (and possibly 6 ) for $\Lambda=947 \mathrm{~nm}$ and to $q=6-10$ for $\Lambda=1610 \mathrm{~nm}$, as observed in the experiment. In addition, we notice that the observed period $d=150-280 \mathrm{~nm}$ in the self-organized nanostructures shown in Fig. 4 is consistent with $D$ at $\varepsilon^{*}<0$ in Fig. 9. It is clear that the lack of SPP mode control in the self-organization process results in the non-uniform period in the nanostructures.

The nanograting period was observed to increase with increasing $F$ (Figs.6 and 8). This can be explained as follows by taking into account a spatial gradient of $N_{\mathrm{e}}$ and the skin depth $\delta$ in the excited layer $b$. The ablation rate $\eta$ in the second step may be estimated as $\eta=20-$ $25 \mathrm{~nm}$ per pulse from the valley depth $h(\sim 700 \mathrm{~nm})$ produced with $N=30-40$. This ablation rate $\eta$ should be comparable to or smaller than $\delta$ in the excited layer $b$. The calculated result in Fig. 9 shows that $\delta=25-50 \mathrm{~nm}(>\eta)$ is achieved only in the threshold region of $\varepsilon^{*}<0$ or at $N_{\mathrm{e}} \approx(2-4) \times 10^{21} \mathrm{~cm}^{-3}$, suggesting that the ablation for the nanograting formation predominantly takes place in this region of $N_{\mathrm{e}}$. Since a higher fluence should more strongly localize the charges in the excited layer $b$, the periodically enhanced near-fields in SPPs exceed $F_{\text {th }}$ in the deeper surface layer with a lower value of $N_{\mathrm{e}}$. As seen in in Fig. 9, the period $D$ to be created increases with decreasing $N_{\mathrm{e}}$ in the threshold region of $\varepsilon^{*}<0$ for the interface $b / c$. This is consistent with the observation of a larger value of $d$ at the higher fluence, as well as the larger ablation rate.

\section{Summary}

We have investigated the fundamental mechanism of the periodic surface nanostructure formation on solid surfaces irradiated with superimposed multiple shots of fs laser pulses at lower fluence than the single pulse ablation threshold. It is shown that the nanostructuring with an increase in $N$ at the low fluence develops through the bonding structure change, the near-field ablation, and the excitation of SPPs, where the non-thermal interaction process is 
crucial to maintain the nanoscale energy distribution in the surface layer until the onset of ablation. Using a simple two-step ablation process, we have demonstrated that the periodic nanostructure formation can be controlled to fabricate a homogeneous nanograting on $\mathrm{GaN}$ surface in air. The experimental results obtained represent exactly the nature of a single standing SPP wave mode for the formation of a homogeneous nanograting. The model calculation has reproduced well the nanograting period and explained the characteristic properties observed in the experiment.

Acknowledgements This work was partially supported by the Grant-in-Aid for Scientific Research 23360034, 24686011, and 22110709.

\section{References}

1. F. Korte, J. Serbin, J. Koch, A. Egbert, C. Fallnich, A. Ostendorf, B. N. Chichkov, Appl. Phys. A 77, 229 (2003)

2. K. Sugioka, M. Meunier, A. Pique (eds.), Laser Precision Microfabrication (Springer, Berlin, 2010)

3. J. Reif, F. Costache, M. Henyk, S. V. Pandelov, Appl. Surf. Sci. 197-198, 891 (2002)

4. N. Yasumaru, K. Miyazaki, J. Kiuchi, Appl. Phys. A 76, 983 (2003)

5. Q. Wu, Y. Ma, R. Fang, Y. Liao, Q. Yu, X. Chen, K. Wang, Appl. Phys. Lett. 82, 1703 (2003)

6. Y. Dong, P. Molian, Appl. Phys. Lett. 84, 10 (2004)

7. A. Borowiec, H. K. Haugen, Appl. Phys. Lett. 82, 4462 (2003)

8. G. Daminelli, J. Krüger, and W.Kautek, Thin Solid Films 467, 334 (2004)

9. C. Wang, H. Huo, M. Johnson, M. Shen, E. Mazur, Nanotechnology 21, 075304 (2010)

10. Q. Z. Zhao, S. Malzer, and L. J. Wang, Opt. Lett. 32, 1932 (2007)

11. E. V. Golosov, V. I. Emel'yanov, A. A. Ionin, Yu. R. Kolobov, S. I. Kudryashov, A. E. Ligachev, Yu. N. Novoselov, L. V. Seleznev, D. V. Sinitsyn, JETP Lett. 90, 107 (2009)

12. N. Yasumaru, E. Sentoku, K. Miyazaki, J. Kiuchi, Appl. Sci. Sci. 264, 611 (2013)

13. J. E. Sipe, J. F. Young, J. S. Preston, H.M. van Driel, Phys. Rev. B 27, 1141 (1983)

14. A. E. Siegman, P. M. Faucher, IEEE J. Quantum Electron. QE-22, 1384 (1986), and references therein

15. J. Bonse, A. Rosenfeld, J. Krüger, J. Appl. Phys. 106, 104910 (2009)

16. M. Huang, F. Zhao, Y. Cheng, N. Xu, Z. Xu, ACS Nano 3, 4062 (2009) 
17. G. D. Tsibidis, M. Barberoglou, P. A. Loukakos, E. Stratakis, C. Fotakis, Phys. Rev. B 86, $115316(2012)$

18. O. Varlamova, J. Reif, S. Varlamov, M. Bestehorn, Appl. Surf. Sci. 257, 5465 (2011)

19. R. Le Harzic, F. Stracke, H. Zimmermann, J. Appl. Phys. 113, 183503 (2013)

20. R. Buividas, L. Rosa, R. Sliupas, T. Kudrius, G. Slekys, V. Datsyuk, S. Juodkazis, Nanotechnology 22, 055304 (2011)

21. V. R. Bhardwaj, E. Simova, P. P. Rajeev, C. Hnatovsky, R. S. Taylor, D. M. Rayner, P. B. Corkum, Phys. Rev. Lett. 96, 057404 (2006)

22. G. Miyaji, K. Miyazaki, Appl. Phys. Lett. 89, 191902 (2006)

23. G. Miyaji, K. Miyazaki, Appl. Phys. Lett. 91, 123102 (2007)

24. G. Miyaji, K. Miyazaki, Opt. Express 16, 16265 (2008)

25. G. Miyaji, K. Miyazaki, K. Zhang, T. Yoshifuji, J. Fujita, Opt. Express 20, 14848 (2012)

26. K. Miyazaki, Nanostructuring of thin film surfaces in femtosecond laser ablation, in Nanophotonics and Nanofabrication, ed. M. Ohtsu (Wiley-VCH, Weinheim, 2009), p. 193

27. K. Miyazaki, N. Maekawa, W. Kobayashi, M. Kaku, N. Yasumaru, J. Kiuchi, Appl. Phys. A 80, 17 (2005)

28. G. Miyaji, K. Miyazaki, Appl. Phys. Lett. 103, 071910(2013)

29. K.Miyazaki, G. Miyaji, J. Appl. Phys. 114, 153108 (2013)

30. G. Miyaji, K. Miyazaki, SPIE Newsroom 24 Oct. 2012, DOI: 10.1117/2.1201210.004516

31. K. Miyazaki, G. Miyaji, Phys. Precedia 39, 674 (2012)

32. N. Yasumaru, K. Miyazaki, J. Kiuchi, Appl. Phys. A 79, 425 (2004)

33. D. von der Linde, K. Sokolowski-Tinten, J. Bialkowski, Appl. Surf. Sci. 109/110, 1 (1997)

34. S. K. Sundaram, E. Mazur, Nat. Mat. 1, 217 (2002)

35. J. Bonse, S. Baudach, J. Krüger, W. Kautek, M. Lenzner, Appl. Phys. A 74, 19 (2002)

36. H. Raether, Surface Plasmons on Smooth and Rough Surfaces and on Gratings (Springer, Berlin, 1988)

37. W. L. Barnes, S. C. Kitson, T. W. Preist, and J. R. Samples, J. Opt. Soc. Am. A 14, 1654 (1997)

38. F. Korte, S. Adams, A. Egbert, C. Fallnich, A. Ostendorf, S. Nolte, M. Will, J. -P. Ruske, B. N.

Chichkov, A. Tünnermann, Opt. Express 7, 41 (2000)

39. C.V. Shank, R. Yen, C. Hirlimann, Phys. Rev. Lett. 50, 454 (1983)

40. M. C. Downer, C.V. Shank, Phys. Rev. Lett. 56, 761 (1986) 
41. G. Yu, G. Wang, H. Ishikawa, M. Umeno, T. Soga, T. Egawa, J. Watanabe, T. Jimbo, Appl. Phys. Lett. 70, 3209 (1997)

42. K. Sokolowski-Tinten, D. von der Linde, Phys. Rev. B 61, 2643 (2000)

43. M. Suzuki, T. Uenoyama, Jpn. J. Appl. Phys. 34, 3442 (1995) 


\section{FIGURE CAPTIONS}

Fig. 1 SEM images (upper) of DLC surface irradiated with superimposed multiple shots (a) $N=300$, (b) $N=500$, and (c) $N=1000$ of fs laser pulses at a fluence of $F<F_{1}$, and schematic of the interaction process (lower) to create the surface structure. The laser polarization direction is horizontal.

Fig. 2 (a) Optical configuration for the pump-probe measurement of the reflectivity $\eta$ of Si surface in water, (b) the temporal change of $\eta$ observed with a single fs laser pulse, and (c) $\eta$ measured as a function of the superimposed number $N$ of fs laser pulses at the time delay of $\Delta t=0.5 \mathrm{ps} . \quad F=120 \mathrm{~mJ} / \mathrm{cm}^{2}$ in (b) and (c).

Fig. 3 Temporal change in the peak reflectivity $\eta_{\text {max }}$ observed at $F_{\text {low }}=120 \mathrm{~mJ} / \mathrm{cm}^{2}$ (solid circles) and $F_{\text {high }}=160 \mathrm{~mJ} / \mathrm{cm}^{2}$ (open circles) for Si immersed in water.

Fig. 4 SEM image of self-organized periodic nanostructure and the period distribution observed for GaN with $N=100$ at (a) $F=400 \mathrm{~mJ} / \mathrm{cm}^{2}$ and (b) $F=480 \mathrm{~mJ} / \mathrm{cm}^{2}$. The fs laser polarization direction is horizontal.

Fig. 5 (a) Schematic of optical configuration for the two-step ablation process. In the first step, the interference fringes are formed in the direction perpendicular to the polarization of Beam 1 and 2, which are incident at the relative angle $\theta$. Only Beam 1 is used in the second step, while Beam 2 is blocked out in the optical arrangement. (b) SPM image of the interference pattern produced in the first step on $\mathrm{GaN}$ with a single fs pulse from two beams at $\theta=59^{\circ}$. The fringe period is $\Lambda=937 \mathrm{~nm}$ with the grooves of $75 \mathrm{~nm}$ in depth.

Fig. 6 SEM image of GaN surface and frequency spectrum of the periodic structure after irradiation of multiple shots of fs laser pulses (a) $N=0$, (b) $N=10$, (c) $N=20$, and (d) $N=40$ at $F=400 \mathrm{~mJ} / \mathrm{cm}^{2}$ from Beam 1 in the second step. The image area is $5 \times 5 \mu \mathrm{m}^{2}$, and the fs laser polarization direction is horizontal.

Fig. 7 STEM image (lower) of the nanograting (upper SPM image), representing the periodically ablated deep valleys aligned on a smooth horizontal level. 
Fig. 8 SEM image and its frequency spectrum of the surface structure after irradiation with (a) $N=10$ and (b) $N=30$ at $F=480 \mathrm{~mJ} / \mathrm{cm}^{2}$ from Beam 1 in the second step. The initial GaN surface at $N=0$ is the same as that shown in Fig. 6(a). The image area is $5 \times 5 \mu \mathrm{m}^{2}$, and the fs laser polarization direction is horizontal.

Fig. 9 The half period $D=\lambda_{\text {spp }} / 2$ of periodically enhanced SPP fields at the interfaces $a / b$ (blue curve) and $b / c$ (red curve), and the skin depth $\delta$ (black curve), calculated as a function of the electron density $N_{\mathrm{e}}$ in the layer $b$. The excitation of SPPs is allowed in the region of $\varepsilon^{*}<$ 0 with $N_{\mathrm{e}}>1.7 \times 10^{21} \mathrm{~cm}^{-3}$, where the results of $D$ and $\delta$ are depicted with solid curves. The inset represents the model surface for the calculation of the nanograting period in the second step, where the fs laser pulse (arrow) incident in air (layer $a$ ) is assumed to create a thin metallic layer $b$ through ionization of GaN substrate (layer $c$ ). 
Fig. 1, Miyazaki

a

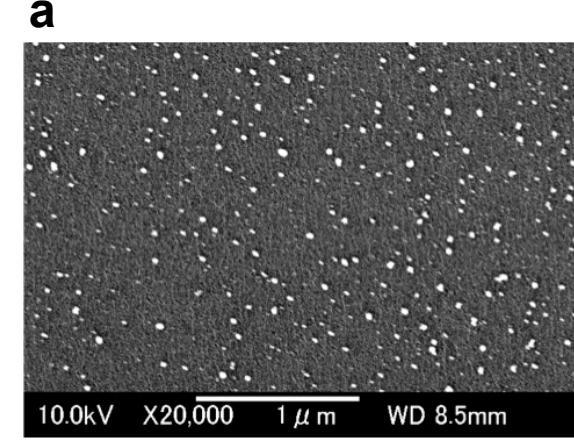

b

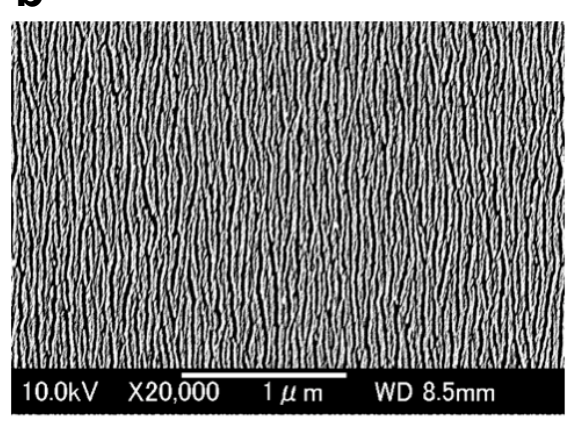

C

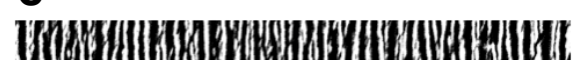

(n)

so

(5)

$10.0 \mathrm{kV} \times 20,000 \quad 1 \mu \mathrm{m}$ WD $8.5 \mathrm{~mm}$

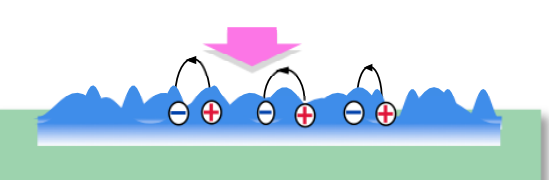


Fig. 2, Miyazaki

a

b

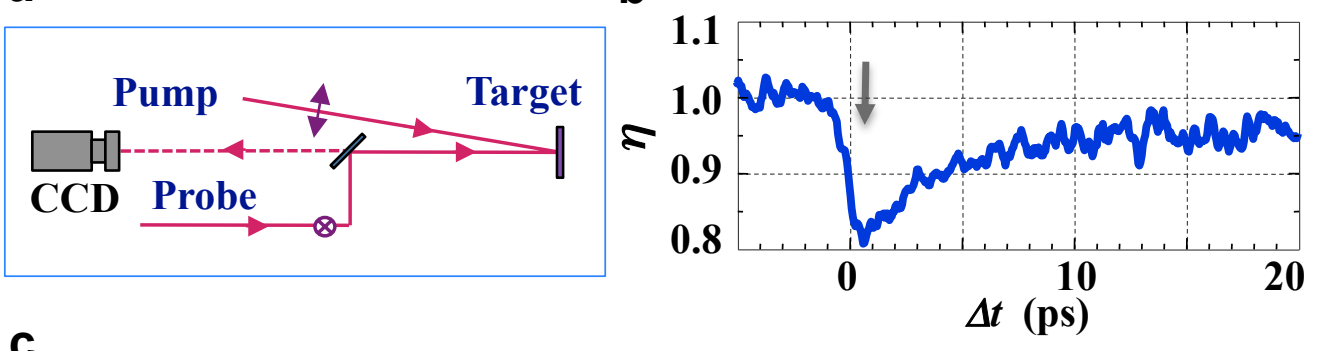

C

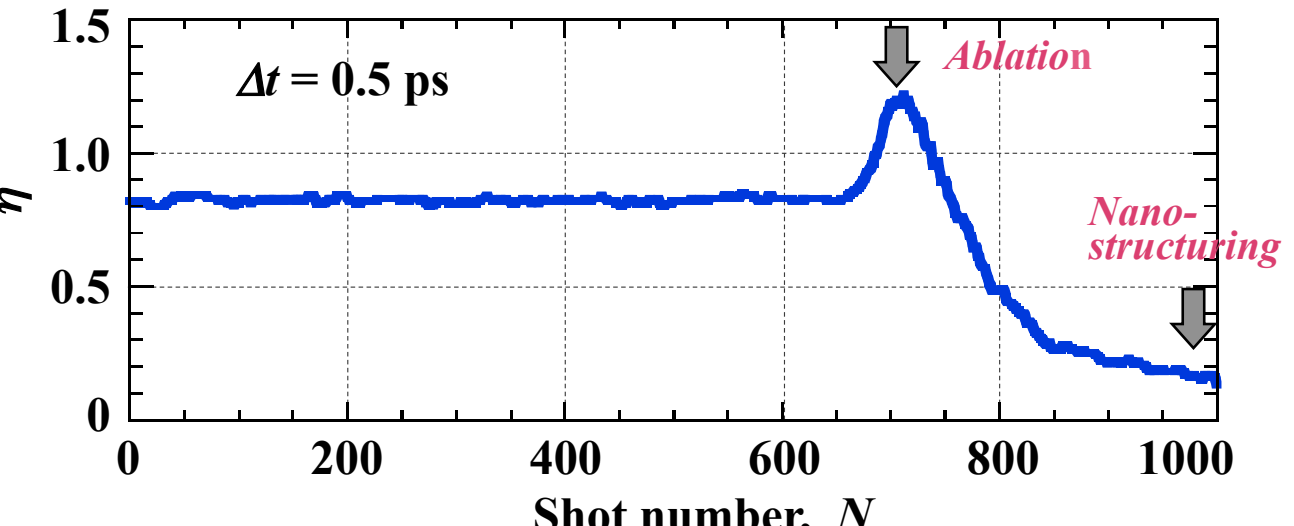




\section{Fig. 3, Miyazaki}

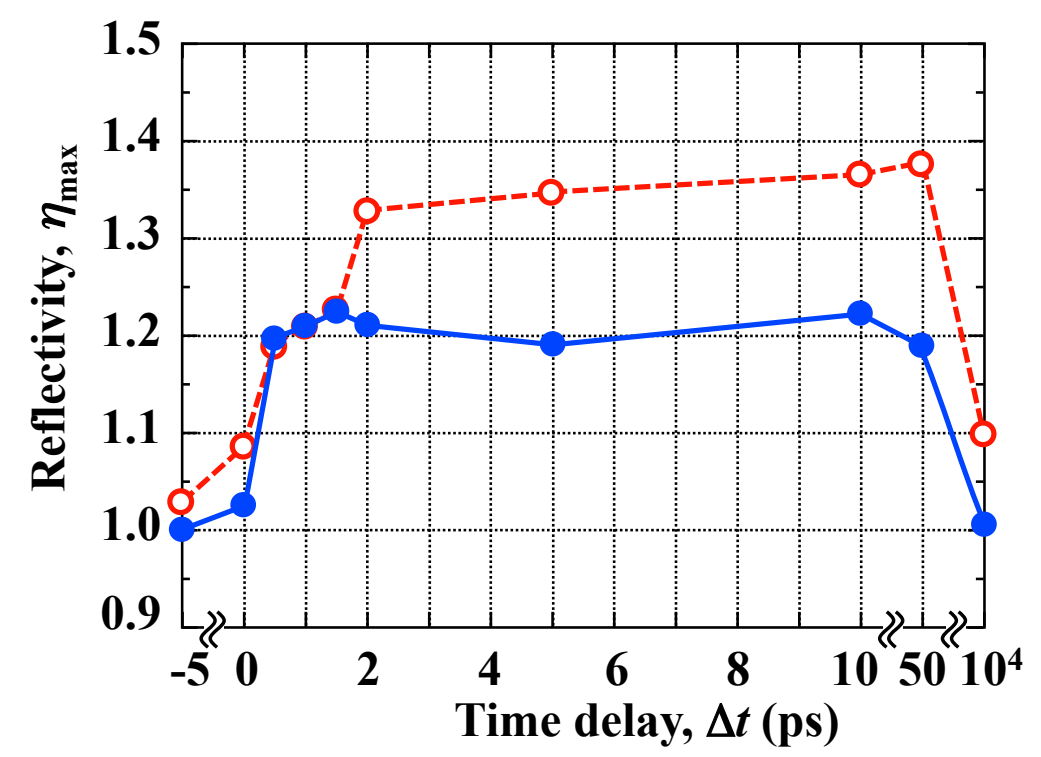


Fig. 4, Miyazaki

a
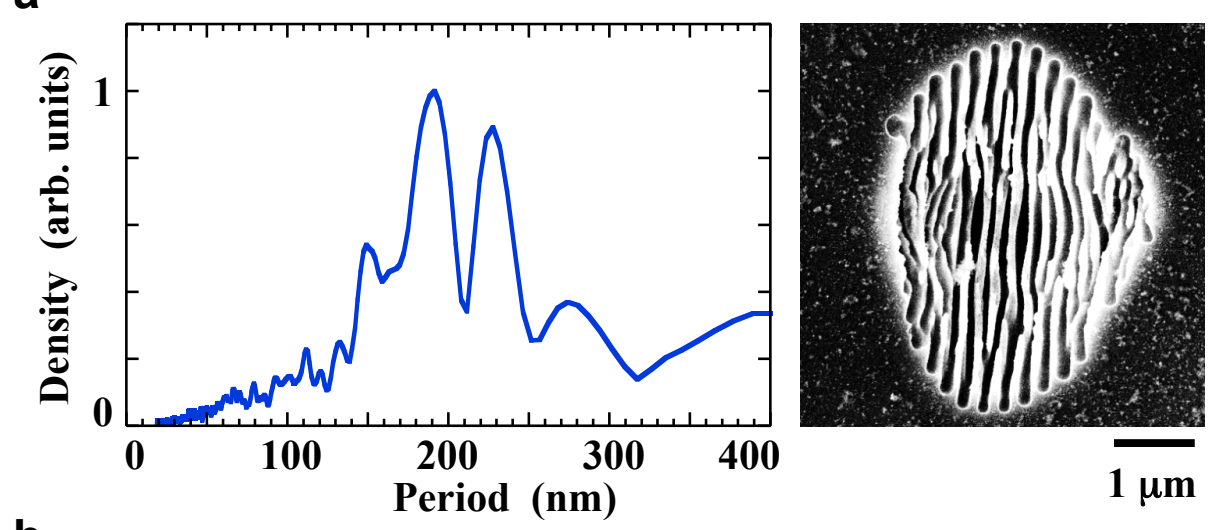

b

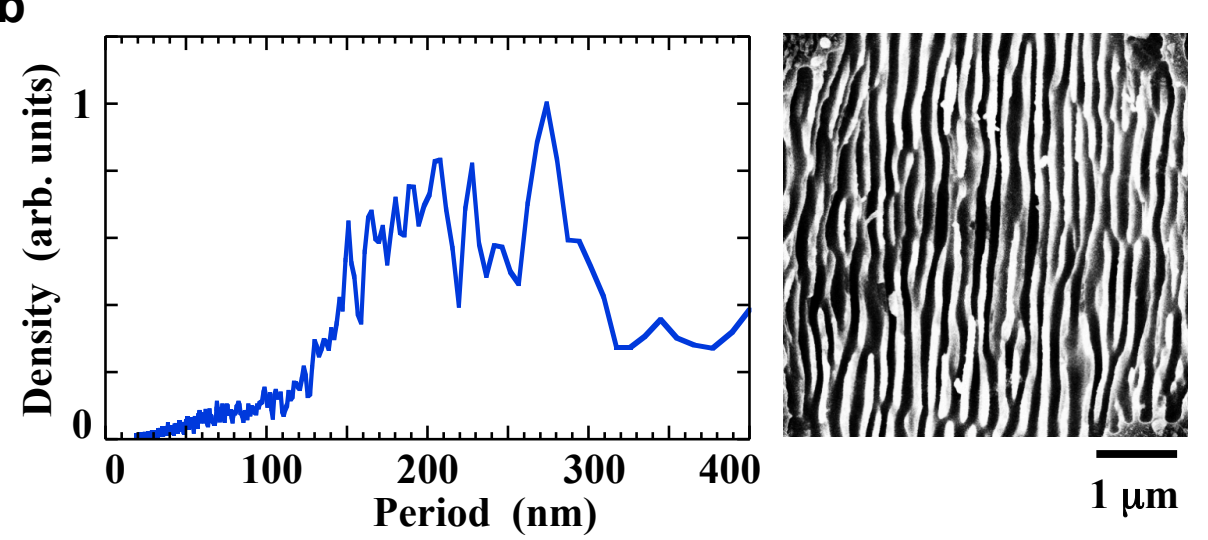


Fig. 5, Miyazaki
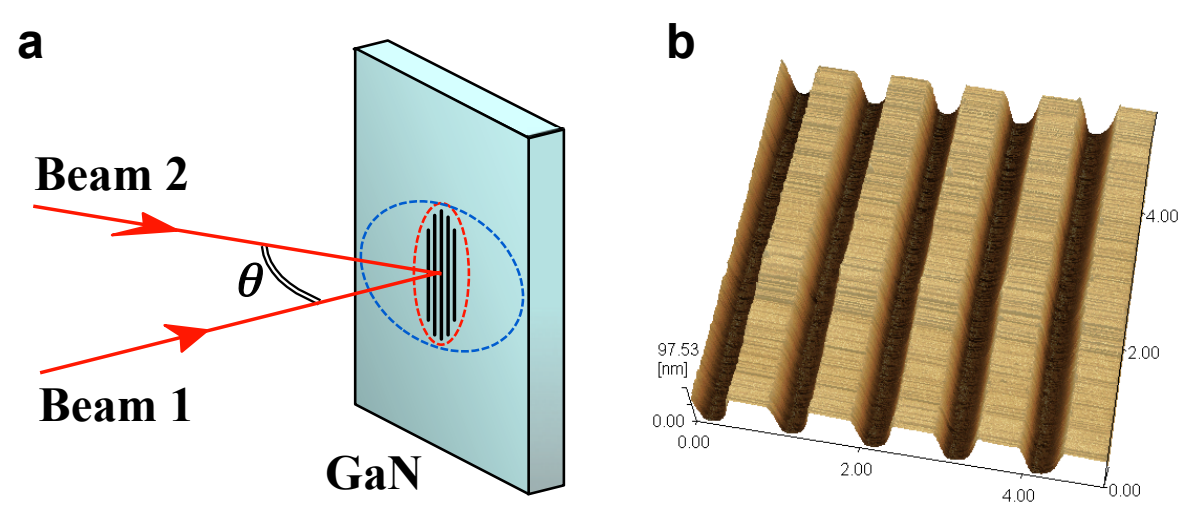
Fig. 6, Miyazaki
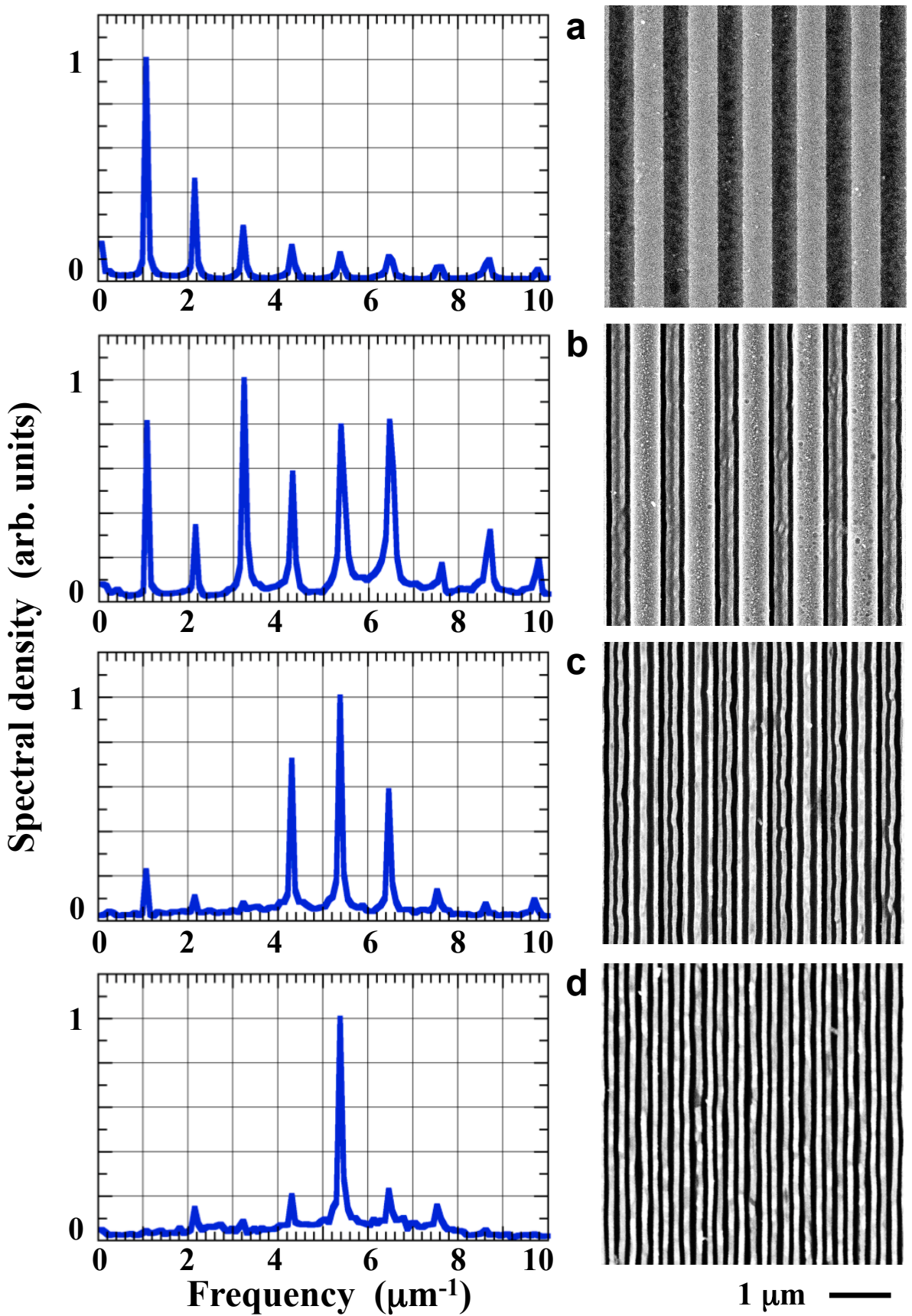
Fig. 7, Miyazaki
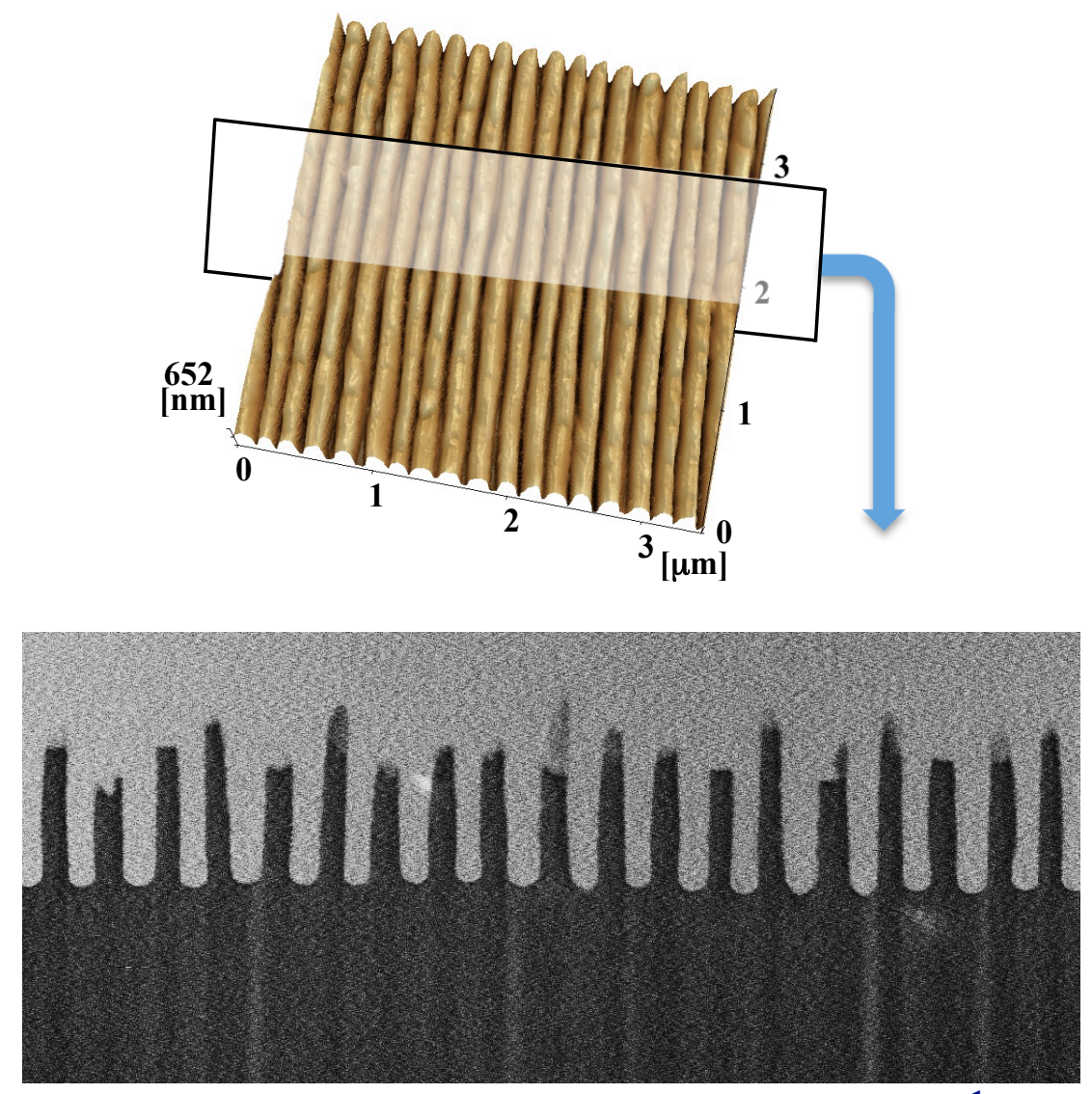

$1 \mu \mathrm{m}$ 
Fig. 8, Miyazaki

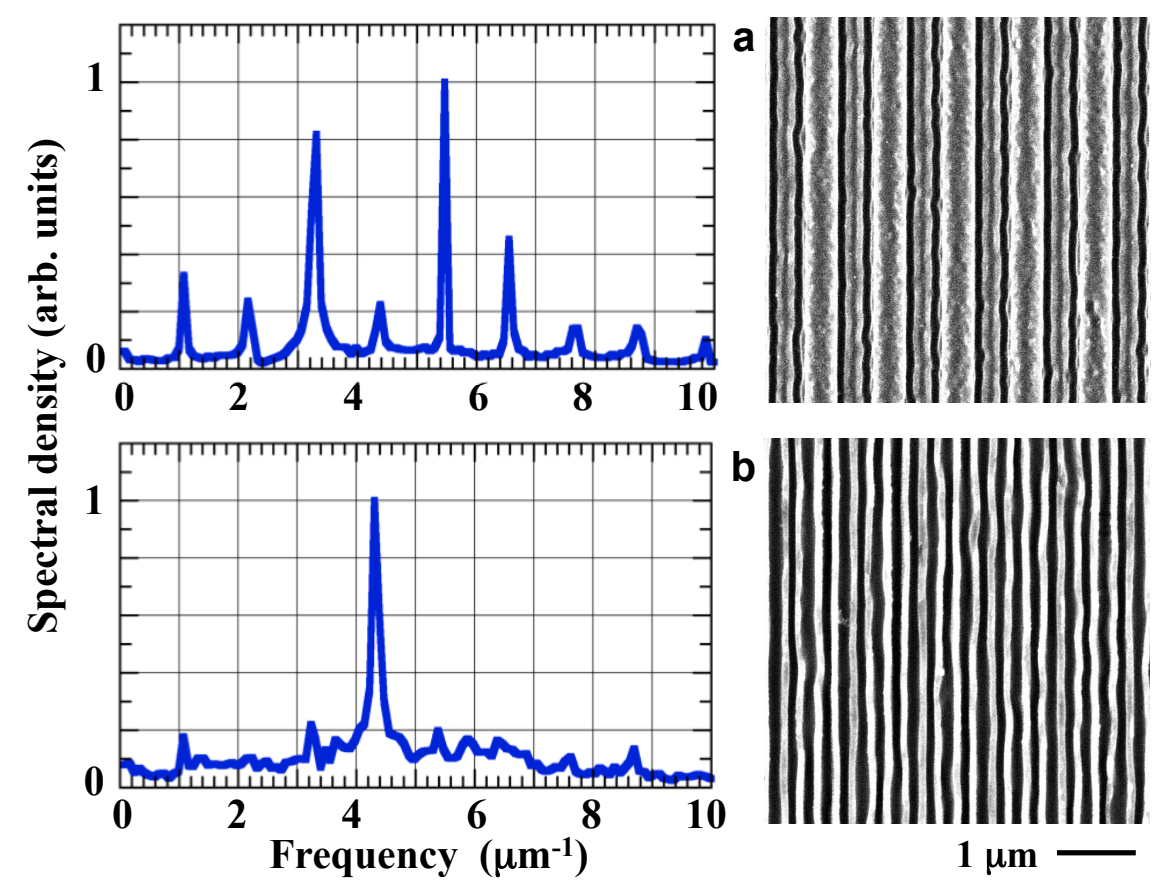


Fig. 9, Miyazaki

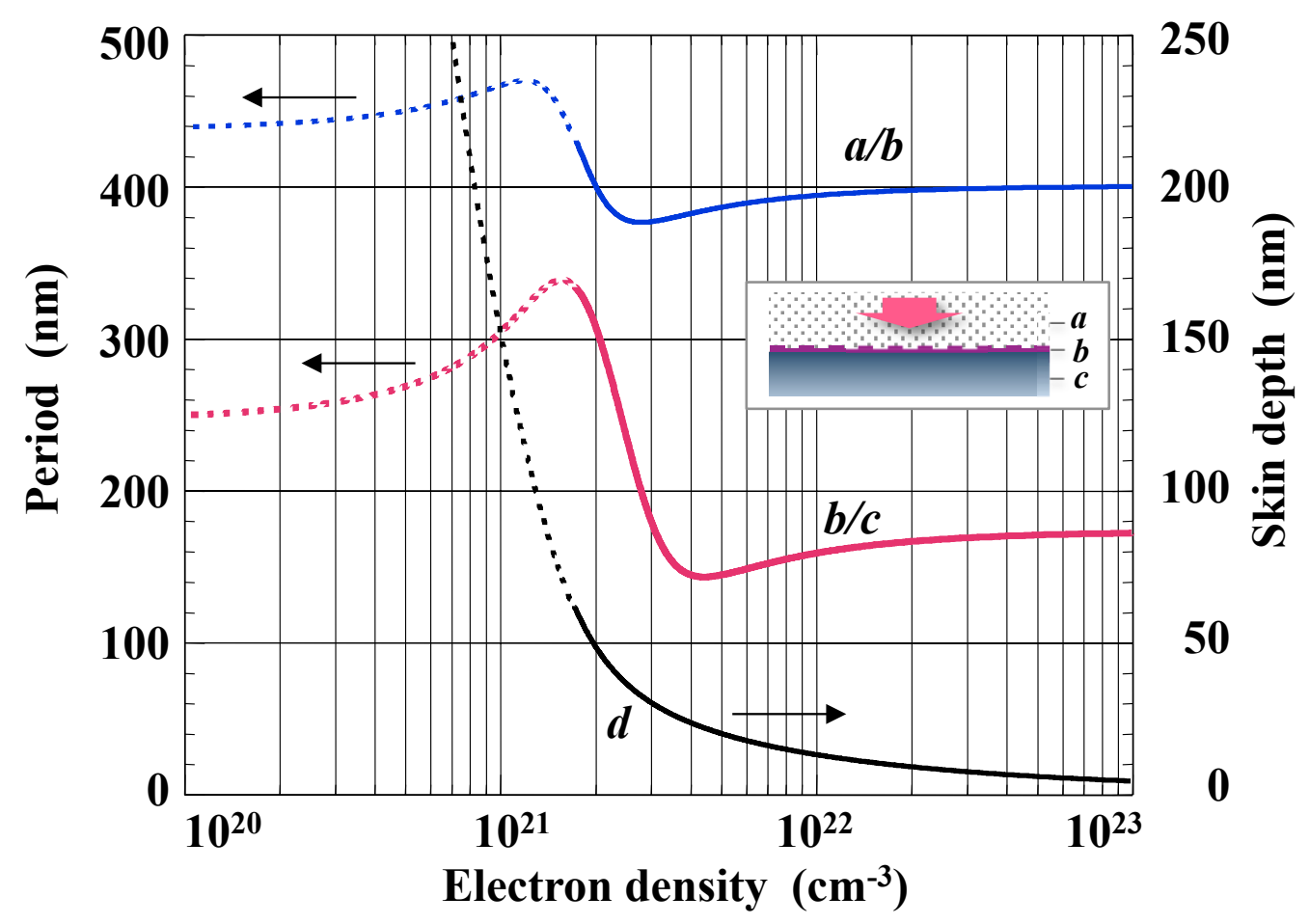

